\title{
Two-Grid $h p$-DGFEM for Second Order Quasilinear Elliptic PDEs Based on an Incomplete Newton Iteration
}

\author{
Scott Congreve and Paul Houston
}

\begin{abstract}
In this paper we propose a class of so-called two-grid $h p$-version discontinuous Galerkin finite element methods for the numerical solution of a second-order quasilinear elliptic boundary value problem based on the application of a single step of a nonlinear Newton solver. We present both the $a$ priori and a posteriori error analysis of this two-grid $h p$-version DGFEM as well as performing numerical experiments to validate the bounds.
\end{abstract}

\section{Introduction}

In our recent articles $[\mathbf{4}, \mathbf{5}]$ we have considered a class of two-grid finite element methods for strongly monotone partial differential equations. Here, the underlying problem is first approximated on a coarse finite element space; the resulting coarse solution is then used to linearise the underlying problem on a finer finite element space, so that only a linear system of equations is solved on this richer space. In this paper we consider an alternative two-grid interior penalty (IP) discontinuous Galerkin finite element method (DGFEM), based on employing a single step of a Newton solver on the finer space, cf. [1] , [9, Section 5.2], for the numerical solution of the following quasilinear elliptic boundary value problem:

$$
-\nabla \cdot(\mu(\boldsymbol{x},|\nabla u|) \nabla u)=f \quad \text { in } \Omega, \quad u=0 \quad \text { on } \Gamma,
$$

where $\Omega$ is a bounded polygonal domain in $\mathbb{R}^{2}$, with boundary $\Gamma$ and $f \in L^{2}(\Omega)$.

We assume that $\mu \in \mathrm{C}^{2}(\bar{\Omega} \times[0, \infty))$ satisfies the condition: there exists positive constants $m_{\mu}$ and $M_{\mu}$ such that the following monotonicity property is satisfied:

$$
m_{\mu}(t-s) \leq \mu(\boldsymbol{x}, t) t-\mu(\boldsymbol{x}, s) s \leq M_{\mu}(t-s), \quad t \geq s \geq 0, \quad \boldsymbol{x} \in \bar{\Omega} .
$$

For ease of notation we write $\mu(t)$ instead of $\mu(\boldsymbol{x}, t)$. The outline of this article is as follows. In Section 2 we state the proposed two-grid IP DGFEM. In Sections 3 and 4 we consider the a priori and a posteriori error analysis, respectively, of the two-grid IP DGFEM. Finally, in Section 5 we present some numerical results to validate the theoretical error bounds.

2010 Mathematics Subject Classification. Primary 65N12, 65N30, 65N50; Secondary 65N35. $\mathrm{PH}$ acknowledges the financial support of the EPSRC under the grant EP/H005498. 


\section{Two-Grid $h p$-Version IP DGFEM}

We consider shape-regular meshes $\mathcal{T}_{h}$ that partition $\Omega \subset \mathbb{R}^{2}$ into open disjoint elements $\kappa$ such that $\bar{\Omega}=\bigcup_{\kappa \in \mathcal{T}_{h}} \bar{\kappa}$. By $h_{\kappa}$ we denote the element diameter of $\kappa \in \mathcal{T}_{h}, h=\max _{\kappa \in \mathcal{T}_{h}} h_{\kappa}$, and $\boldsymbol{n}_{\kappa}$ signifies the unit outward normal vector to $\kappa$. We allow the meshes $\mathcal{T}_{h}$ to be 1-irregular; further, we suppose that $\mathcal{T}_{h}$ is of bounded local variation, i.e., there exists a constant $\rho_{1} \geq 1$, independent of the element sizes, such that $\rho_{1}^{-1} \leq h_{\kappa} / h_{\kappa^{\prime}} \leq \rho_{1}$, for any pair of elements $\kappa, \kappa^{\prime} \in \mathcal{T}_{h}$ which share a common edge $e=\partial \kappa \cap \partial \kappa^{\prime}$. To each $\kappa \in \mathcal{T}_{h}$ we assign a polynomial degree $p_{\kappa} \geq 1$ and define the degree vector $\boldsymbol{p}=\left\{p_{\kappa}: \kappa \in \mathcal{T}_{h}\right\}$. We suppose that $\boldsymbol{p}$ is also of bounded local variation, i.e., there exists a constant $\rho_{2} \geq 1$, independent of the element sizes and $\boldsymbol{p}$, such that, for any pair of neighbouring elements $\kappa, \kappa^{\prime} \in \mathcal{T}_{h}, \rho_{2}^{-1} \leq p_{\kappa} / p_{\kappa^{\prime}} \leq \rho_{2}$.

With this notation, we introduce the finite element space

$$
V\left(\mathcal{T}_{h}, \boldsymbol{p}\right)=\left\{v \in L^{2}(\Omega):\left.v\right|_{\kappa} \in \mathcal{S}_{p_{\kappa}}(\kappa) \quad \forall \kappa \in \mathcal{T}_{h}\right\},
$$

where $\mathcal{S}_{p_{\kappa}}(\kappa)=\mathcal{P}_{p_{\kappa}}(\kappa)$ if $\kappa$ is a triangle and $\mathcal{S}_{p_{\kappa}}(\kappa)=\mathcal{Q}_{p_{\kappa}}(\kappa)$ if $\kappa$ is a parallelogram. Here, for $p \geq 0, \mathcal{P}_{p}(\kappa)$ denotes the space of polynomials of degree at most $p$ on $\kappa$, while $\mathcal{Q}_{p}(\kappa)$ is the space of polynomials of degree at most $p$ in each variable on $\kappa$.

For the mesh $\mathcal{T}_{h}$, we write $\mathcal{E}_{h}^{\mathcal{I}}$ to denote the set of all interior edges of the partition $\mathcal{T}_{h}$ of $\Omega, \mathcal{E}_{h}^{\mathcal{B}}$ the set of all boundary edges of $\mathcal{T}_{h}$, and set $\mathcal{E}_{h}=\mathcal{E}_{h}^{\mathcal{B}} \cup \mathcal{E}_{h}^{\mathcal{I}}$. Let $v$ and $\boldsymbol{q}$ be scalar- and vector-valued functions, respectively, which are sufficiently smooth inside each element $\kappa \in \mathcal{T}_{h}$. Given two adjacent elements, $\kappa^{+}, \kappa^{-} \in \mathcal{T}_{h}$ which share a common edge $e \in \mathcal{E}_{h}^{\mathcal{I}}$, i.e., $e=\partial \kappa^{+} \cap \partial \kappa^{-}$, we write $v^{ \pm}$and $\boldsymbol{q}^{ \pm}$ to denote the traces of the functions $v$ and $\boldsymbol{q}$, respectively, on the edge $e$, taken from the interior of $\kappa^{ \pm}$, respectively. With this notation, the averages of $v$ and $\boldsymbol{q}$ at $\boldsymbol{x} \in e$ are given by $\left\{\{v\}=1 / 2\left(v^{+}+v^{-}\right)\right.$and $\left\{\{\boldsymbol{q}\}=1 / 2\left(\boldsymbol{q}^{+}+\boldsymbol{q}^{-}\right)\right.$, respectively. Similarly, the jumps of $v$ and $\boldsymbol{q}$ at $\boldsymbol{x} \in e$ are given by $\llbracket v \rrbracket=v^{+} \boldsymbol{n}_{\kappa^{+}}+v_{\kappa^{-}}^{-} \boldsymbol{n}_{\kappa^{-}}$and $\llbracket \boldsymbol{q} \rrbracket=\boldsymbol{q}^{+} \cdot \boldsymbol{n}_{\kappa^{+}}+\boldsymbol{q}^{-} \cdot \boldsymbol{n}_{\kappa^{-}}$, respectively, where $\boldsymbol{n}_{\kappa^{ \pm}}$denotes the unit outward normal vector on $\partial \kappa^{ \pm}$, respectively. On a boundary edge $e \in \mathcal{E}_{h}^{\mathcal{B}}$, we set $\{\{v\}=v,\{\{\boldsymbol{q}\}\}=\boldsymbol{q}$, $\llbracket v \rrbracket=v \boldsymbol{n}$ and $\llbracket \boldsymbol{q} \rrbracket=\boldsymbol{q} \cdot \boldsymbol{n}$, with $\boldsymbol{n}$ denoting the unit outward normal vector on the boundary $\Gamma$. For $e \in \mathcal{E}_{h}$, we define $h_{e}$ to be the length of the edge; moreover, we set $p_{e}=\max \left(p_{\kappa}, p_{\kappa^{\prime}}\right)$, if $e=\partial \kappa \cap \partial \kappa^{\prime} \in \mathcal{E}_{h}^{\mathcal{I}}$, and $p_{e}=p_{\kappa}$, if $e=\partial \kappa \cap \Gamma \in \mathcal{E}_{h}^{\mathcal{B}}$.

2.1. Standard IP DGFEM discretisation. Given a fine mesh partition $\mathcal{T}_{h}$ of $\Omega$, with the corresponding polynomial degree vector $\boldsymbol{p}$, the standard IP DGFEM is defined as follows: find $u_{h, p} \in V\left(\mathcal{T}_{h}, \boldsymbol{p}\right)$ such that

$$
A_{h, p}\left(u_{h, p}, v_{h, p}\right)=F_{h, p}\left(v_{h, p}\right)
$$

for all $v_{h, p} \in V\left(\mathcal{T}_{h}, \boldsymbol{p}\right)$, where $F_{h, p}(v)=\int_{\Omega} f v \mathrm{~d} \boldsymbol{x}$ and

$$
\begin{aligned}
A_{h, p}(u, v)= & \int_{\Omega} \mu\left(\left|\nabla_{h} u\right|\right) \nabla_{h} u \cdot \nabla_{h} v \mathrm{~d} \boldsymbol{x}-\sum_{e \in \mathcal{E}_{h}} \int_{e}\left\{\mu\left(\left|\nabla_{h} u\right|\right) \nabla_{h} u\right\} \cdot \llbracket v \rrbracket \mathrm{d} s \\
& \left.+\theta \sum_{e \in \mathcal{E}_{h}} \int_{e}\left\{\mu\left(h_{e}^{-1}|\llbracket u \rrbracket|\right) \nabla_{h} v\right\}\right\} \cdot \llbracket u \rrbracket \mathrm{d} s+\sum_{e \in \mathcal{E}_{h}} \int_{e} \sigma_{h, p} \llbracket u \rrbracket \cdot \llbracket v \rrbracket \mathrm{d} s .
\end{aligned}
$$

Here, $\theta \in[-1,1], \nabla_{h}$ is the element-wise gradient operator and $\sigma_{h, p}=\gamma p_{e}^{2} / h_{e}$, where $\gamma>0$ is a sufficiently large constant. We define the energy norm on $V\left(\mathcal{T}_{h}, \boldsymbol{p}\right)$ :

$$
\|v\|_{\mathrm{DG}}^{2}=\left\|\nabla_{h} v\right\|_{L^{2}(\Omega)}^{2}+\sum_{e \in \mathcal{E}_{h}} \int_{e} \sigma_{h, p}|\llbracket v \rrbracket|^{2} \mathrm{~d} s .
$$


LEMMA 2.1 (See $[6]$ ). The semilinear form $A_{h, p}(\cdot, \cdot)$ is strongly monotone in the sense that, there exists $\gamma_{\min }>0$, such that for any $\gamma \geq \gamma_{\min }$ $(2.2)$

$$
A_{h, p}\left(w_{1}, w_{1}-w_{2}\right)-A_{h, p}\left(w_{2}, w_{1}-w_{2}\right) \geq C_{m}\left\|w_{1}-w_{2}\right\|_{\mathrm{DG}}^{2} \forall w_{1}, w_{2} \in V\left(\mathcal{T}_{h}, \boldsymbol{p}\right),
$$

where $C_{m}$ is a positive constant, independent of the discretisation parameters.

2.2. Two-grid IP DGFEM discretisation. We now introduce a two-grid IP DGFEM based on employing a single step of the Newton iteration on the fine mesh. To this end, we consider two partitions $\mathcal{T}_{h}$ and $\mathcal{T}_{H}$ of $\Omega$, with granularity $h$ and $H$, respectively. We assume that $\mathcal{T}_{h}$ and $\mathcal{T}_{H}$ are nested in that sense that for any element $\kappa_{h} \in \mathcal{T}_{h}$ there exists an element $\kappa_{H} \in \mathcal{T}_{H}$ such that $\bar{\kappa}_{h} \subseteq \bar{\kappa}_{H}$. Moreover for each mesh, $\mathcal{T}_{h}$ and $\mathcal{T}_{H}$, we have a corresponding polynomial degree vector $\boldsymbol{p}=$ $\left\{p_{\kappa}: \kappa \in \mathcal{T}_{h}\right\}$ and $\boldsymbol{P}=\left\{p_{\kappa}: \kappa \in \mathcal{T}_{H}\right\}$, respectively, where given an element $\kappa_{h} \in \mathcal{T}_{h}$ and an element $\kappa_{H} \in \mathcal{T}_{H}$, such that $\bar{\kappa}_{h} \subseteq \bar{\kappa}_{H}$, the polynomial degree vectors satisfy the condition that $p_{\kappa_{h}} \geq p_{\kappa_{H}}$. Thereby, the finite element spaces $V\left(\mathcal{T}_{h}, \boldsymbol{p}\right)$ and $V\left(\mathcal{T}_{H}, \boldsymbol{P}\right)$ satisfy the following the condition: $V\left(\mathcal{T}_{H}, \boldsymbol{P}\right) \subseteq V\left(\mathcal{T}_{h}, \boldsymbol{p}\right)$.

Using this notation we introduce the $h p$-version two-grid IP DGFEM discretisation of (1.1) based on a single Newton iteration step, cf. [1], [9, Section 5.2]:

(1) Compute the coarse grid approximation $u_{H, P} \in V\left(\mathcal{T}_{H}, \boldsymbol{P}\right)$ such that

$$
A_{H, P}\left(u_{H, P}, v_{H, P}\right)=F_{H, P}\left(v_{H, P}\right) \quad \text { for all } v_{H, P} \in V\left(\mathcal{T}_{H}, \boldsymbol{P}\right) \text {. }
$$

(2) Determine the fine grid solution $u_{2 G} \in V\left(\mathcal{T}_{h}, \boldsymbol{p}\right)$ such that

$$
\begin{aligned}
& A_{h, p}^{\prime}\left[u_{H, P}\right]\left(u_{2 G}, v_{h, p}\right)=A_{h, p}^{\prime}\left[u_{H, P}\right]\left(u_{H, P}, v_{h, p}\right)-A_{h, p}\left(u_{H, P}, v_{h, p}\right)+F_{h, p}\left(v_{h, p}\right) \\
& \quad \text { for all } v_{h, p} \in V\left(\mathcal{T}_{h}, \boldsymbol{p}\right) .
\end{aligned}
$$

Here, $A_{h, p}^{\prime}[u](\phi, v)$ denotes the Fréchet derivative of $u \rightarrow A_{h, p}(u, v)$, for fixed $v$, evaluated at $u$; thereby, given $\phi$ we have $A_{h, p}^{\prime}[u](\phi, v)=\lim _{t \rightarrow 0} \frac{A_{h, p}(u+t \phi, v)-A_{h, p}(u, v)}{t}$.

REMARK 2.2. For simplicity of presentation, throughout the rest of this article we shall only consider the incomplete IP variation of the DGFEM, i.e., when $\theta=0$.

LEMмa 2.3. Under the assumptions on $\mu$, the following inequality holds:

$$
A_{h, p}^{\prime}[u](v, v) \geq C_{m}\|v\|_{\mathrm{DG}}^{2} \quad \forall u, v \in V\left(\mathcal{T}_{h}, \boldsymbol{p}\right) .
$$

Proof. Setting $w_{1}=u+t v$ and $w_{2}=u$ in Lemma 2.1, $u, v \in V\left(\mathcal{T}_{h}, \boldsymbol{p}\right), t>0$ :

$$
\frac{A_{h, p}(u+t v, v)-A_{h, p}(u, v)}{t} \geq C_{m}\|v\|_{\mathrm{DG}}^{2} .
$$

Taking the limit as $t \rightarrow 0$, we deduce the statement of the Lemma.

\section{A Priori Error Analysis}

For simplicity of presentation, in this section we assume that the mesh is quasiuniform with mesh size $h$ and that $\boldsymbol{p}$ is uniform over the mesh, i.e., $\boldsymbol{p} \equiv p$.

THEOREM 3.1. Assuming that $u \in C^{1}(\Omega)$ and $u \in H^{k}(\Omega), k \geq 2$, the solution of $u_{2 G} \in V\left(\mathcal{T}_{h}, \boldsymbol{p}\right)$ of the two-grid IP DGFEM satisfies

$$
\begin{aligned}
\left\|u_{h, p}-u_{2 G}\right\|_{\mathrm{DG}} & \leq C \frac{p^{7 / 2}}{h} \frac{H^{2 S-2}}{P^{2 k-3}}\|u\|_{H^{k}(\Omega)}^{2}, \\
\left\|u-u_{2 G}\right\|_{\mathrm{DG}} & \leq C \frac{h^{s-1}}{p^{k-3 / 2}}\|u\|_{H^{k}(\Omega)}+C \frac{p^{7 / 2}}{h} \frac{H^{2 S-2}}{P^{2 k-3}}\|u\|_{H^{k}(\Omega)}^{2},
\end{aligned}
$$


with $1 \leq s \leq \min \{p+1, k\}, p \geq 1$ and $1 \leq S \leq \min \{P+1, k\}, P \geq 1$, where $C>0$ is independent of the discretisation parameters.

3.1. Auxiliary Results. We first state the following auxiliary results.

Lemma 3.2. For a function $v \in V\left(\mathcal{T}_{h}, \boldsymbol{p}\right)$ we have the inverse inequality

$$
\|v\|_{L^{4}(\Omega)} \leq C p h^{-1 / 2}\|v\|_{L^{2}(\Omega)},
$$

where $C$ is a positive constant, independent of the discretisation parameters.

Proof. Given $\kappa \in \mathcal{T}_{h}$, employing standard inverse inequalities, see [8], gives

$$
\int_{\kappa}|v|^{4} \mathrm{~d} \boldsymbol{x} \leq\|v\|_{L^{\infty}(\kappa)}^{2}\|v\|_{L^{2}(\kappa)}^{2} \leq C p^{4} h^{-2}\|v\|_{L^{2}(\kappa)}^{2}\|v\|_{L^{2}(\kappa)}^{2}=C p^{4} h^{-2}\|v\|_{L^{2}(\kappa)}^{4} .
$$

Summing over $\kappa \in \mathcal{T}_{h}$, employing the inequality $\sum_{i=1}^{n} a_{i} \leq\left(\sum_{i=1}^{n} \sqrt{a_{i}}\right)^{2}, a_{i} \geq 0$, $i=1, \ldots, n$, and taking the fourth root of both sides, completes the proof.

Lemma 3.3. For any $v, w, \phi \in V\left(\mathcal{T}_{h}, \boldsymbol{p}\right)$,

$$
A_{h, p}(w, \phi)=A_{h, p}(v, \phi)+A_{h, p}^{\prime}[v](w-v, \phi)+\mathcal{Q}(v, w, \phi),
$$

where the remainder $\mathcal{Q}$ satisfies

$$
|\mathcal{Q}(v, w, \phi)| \leq C p^{2} h^{-1}\left(1+\|\nabla w\|_{L^{\infty}(\Omega)}+\|\nabla v\|_{L^{\infty}(\Omega)}\right)\|\nabla(w-v)\|_{\mathrm{DG}}^{2}\|\nabla \phi\|_{\mathrm{DG}},
$$

and $C$ is a positive constant, independent of the discretisation parameters.

Proof. We follow the proof outlined by [9, Lemma 3.1]; to this end, setting $\xi(t)=v+t(w-v)$ and $\eta(t)=A_{h, p}(\xi(t), \phi)$, we note that the first equation follows from the identity

$$
\eta(1)=\eta(0)+\eta^{\prime}(0)+\int_{0}^{1} \eta^{\prime \prime}(t)(1-t) \mathrm{d} t,
$$

where $\mathcal{Q}(v, w, \phi)=\int_{0}^{1} \eta^{\prime \prime}(t)(1-t) \mathrm{d} t$ and $\eta^{\prime \prime}(t)=A_{h, p}^{\prime \prime}[\xi(t)](w-v, w-v, \phi)$. Thereby,

$$
\begin{aligned}
\mathcal{Q}(v, w, \phi)= & 2 \int_{0}^{1} \int_{\Omega} \mu_{\nabla u}^{\prime}(|\nabla \xi(t)|) \cdot \nabla(w-v) \nabla(w-v) \cdot \nabla \phi \mathrm{d} \boldsymbol{x}(1-t) \mathrm{d} t \\
& +\int_{0}^{1} \int_{\Omega} \mu_{\nabla u}^{\prime \prime}(|\nabla \xi(t)|)|\nabla(w-v)|^{2} \nabla \xi(t) \cdot \nabla \phi \mathrm{d} \boldsymbol{x}(1-t) \mathrm{d} t \\
& -2 \int_{0}^{1} \sum_{e \in \mathcal{E}_{h}} \int_{e}\left\{\left\{\mu_{\nabla u}^{\prime}(|\nabla \xi(t)|) \cdot \nabla(w-v) \nabla(w-v)\right\} \cdot \llbracket \phi \rrbracket \mathrm{d} s(1-t) \mathrm{d} t\right. \\
& -\int_{0}^{1} \sum_{e \in \mathcal{E}_{h}} \int_{e}\left\{\left\{\mu_{\nabla u}^{\prime \prime}(|\nabla \xi(t)|)|\nabla(w-v)|^{2} \nabla \xi(t)\right\} \cdot \llbracket \phi \rrbracket \mathrm{d} s(1-t) \mathrm{d} t\right. \\
\equiv & T_{1}+T_{2}+T_{3}+T_{4} .
\end{aligned}
$$

Here, $\mu_{\nabla u}^{\prime}(|\cdot|)$ and $\mu_{\nabla u}^{\prime \prime}(|\cdot|)$ denote the first and second derivatives of $\mu(|\cdot|)$, respectively. First consider $T_{1}$ : given that $\mu \in \mathrm{C}^{2}(\bar{\Omega} \times[0, \infty))$, Lemma 3.2 gives

$$
T_{1} \leq C\|\nabla(w-v)\|_{L^{4}(\Omega)}^{2}\|\nabla \phi\|_{L^{2}(\Omega)} \leq C p^{2} h^{-1}\|\nabla(w-v)\|_{L^{2}(\Omega)}^{2}\|\nabla \phi\|_{L^{2}(\Omega)} .
$$

Secondly, term $T_{2}$ is bounded in an analogous fashion as follows:

$$
\begin{aligned}
T_{2} & \leq C\left(\|\nabla w\|_{L^{\infty}(\Omega)}+\|\nabla v\|_{L^{\infty}(\Omega)}\right)\|\nabla(w-v)\|_{L^{4}(\Omega)}^{2}\|\nabla \phi\|_{L^{2}(\Omega)} \\
& \leq C\left(\|\nabla w\|_{L^{\infty}(\Omega)}+\|\nabla v\|_{L^{\infty}(\Omega)}\right) p^{2} h^{-1}\|\nabla(w-v)\|_{L^{2}(\Omega)}^{2}\|\nabla \phi\|_{L^{2}(\Omega)} .
\end{aligned}
$$


Term $T_{3}$ is bounded via the inverse trace inequality, see [8], and Lemma 3.2:

$$
\begin{aligned}
T_{3} & \leq C\left\{\sum_{e \in \mathcal{E}_{h}} h_{e} p_{e}^{-2}\left\|\left\{|\nabla(w-v)|^{2}\right\}\right\|_{L^{2}(e)}^{2}\right\}^{\frac{1}{2}}\left\{\sum_{e \in \mathcal{E}_{h}} \int_{F} p_{e}^{2} h_{e}^{-1}|\llbracket \phi \rrbracket|^{2} \mathrm{~d} s\right\}^{\frac{1}{2}} \\
& \leq C\|\nabla(w-v)\|_{L^{4}(\Omega)}^{2}\|\phi\|_{\mathrm{DG}} \leq C p^{2} h^{-1}\|\nabla(w-v)\|_{L^{2}(\Omega)}^{2}\|\phi\|_{\mathrm{DG}} .
\end{aligned}
$$

We can bound $T_{4}$ in an analogous manner as follows:

$$
\begin{aligned}
T_{4} \leq & C\left\{\sum_{e \in \mathcal{E}_{h}} h_{e} p_{e}^{-2} \|\left\{\left\{|\nabla(w-v)|^{2}|\nabla w|\right\} \|_{L^{2}(F)}^{2}\right\}^{\frac{1}{2}}\left\{\sum_{e \in \mathcal{E}_{h}} \int_{F} p^{2} h^{-1}|\llbracket \phi \rrbracket|^{2} \mathrm{~d} s\right\}^{\frac{1}{2}}\right. \\
& +C\left\{\sum_{e \in \mathcal{E}_{h}} h_{e} p_{e}^{-2}\left\|\left\{|\nabla(w-v)|^{2}|\nabla v|\right\}\right\|_{L^{2}(F)}^{2}\right\}^{\frac{1}{2}}\left\{\sum_{e \in \mathcal{E}_{h}} \int_{F} p^{2} h^{-1}|\llbracket \phi \rrbracket|^{2} \mathrm{~d} s\right\}^{\frac{1}{2}} \\
\leq & C\left\{\left\||\nabla(w-v)|^{2}|\nabla w|\right\|_{L^{2}(\Omega)}+\left\||\nabla(w-v)|^{2}|\nabla v|\right\|_{L^{2}(\Omega)}\right\}\|\phi\|_{\mathrm{DG}} \\
\leq & C p^{2} h^{-1}\left\{\|\nabla w\|_{L^{\infty}(\Omega)}+\|\nabla v\|_{L^{\infty}(\Omega)}\right\}\|\nabla(w-v)\|_{L^{2}(\Omega)}^{2}\|\phi\|_{\mathrm{DG}} .
\end{aligned}
$$

Combining these bounds for terms $T_{1}, T_{2}, T_{3}$ and $T_{4}$ completes the proof.

LEMma 3.4. Let $u \in H^{2}(\Omega)$ be the analytical solution of (1.1), such that $\nabla u \in$ $\left[L^{\infty}(\Omega)\right]^{2}$, and $u_{h, p} \in V\left(\mathcal{T}_{h}, \boldsymbol{p}\right)$ be the IP DGFEM defined by (2.1), we have that

$$
\left\|\nabla u_{h, p}\right\|_{L^{\infty}(\Omega)} \leq C p^{3 / 2},
$$

where $C$ is a positive constant, independent of the discretisation parameters.

Proof. Writing $\mathcal{P}_{u}$ to denote the projection of $u$ onto the finite element space $V\left(\mathcal{T}_{h}, \boldsymbol{p}\right)$ defined in [2], we have that $\left\|u-\mathcal{P}_{u}\right\|_{H^{q}(\Omega)} \leq C \frac{h^{2-q}}{p^{2-q}}\|u\|_{H^{2}(\Omega)}$ and $\left\|\nabla\left(u-\mathcal{P}_{u}\right)\right\|_{L^{\infty}(\Omega)} \leq C\|u\|_{H^{2}(\Omega)}$ for all $q \leq 2$. Exploiting these bounds, standard inverse inequalities, $[\mathbf{8}]$, and the a priori bound for the IP DGFEM, [6], gives

$$
\begin{aligned}
\left\|\nabla u_{h, p}\right\|_{L^{\infty}(\Omega)} & \leq\left\|\nabla\left(u_{h, p}-\mathcal{P}_{u}\right)\right\|_{L^{\infty}(\Omega)}+\left\|\nabla \mathcal{P}_{u}\right\|_{L^{\infty}(\Omega)} \\
& \leq C p^{2} h^{-1}\left\|\nabla\left(u_{h, p}-\mathcal{P}_{u}\right)\right\|_{L^{2}(\Omega)}+\left\|\nabla\left(u-\mathcal{P}_{u}\right)\right\|_{L^{\infty}(\Omega)}+\|\nabla u\|_{L^{\infty}(\Omega)} \\
& \leq C p^{3 / 2}\left\{\|u\|_{H^{2}(\Omega)}+\|\nabla u\|_{L^{\infty}(\Omega)}\right\} .
\end{aligned}
$$

Since $u \in H^{2}(\Omega)$ and $\nabla u \in\left[L^{\infty}(\Omega)\right]^{2}$, the quantities $\|u\|_{H^{2}(\Omega)}$ and $\|\nabla u\|_{L^{\infty}(\Omega)}$ are both bounded uniformly by a constant; this then completes the proof.

3.2. Proof of Theorem 3.1. We now exploit the above results to prove Theorem 3.1. For the first bound (3.1), we employ Lemma 2.3, (2.1), (2.4) and (3.3); thereby, with $\phi=u_{h, p}-u_{2 G}$, we deduce that

$$
\begin{aligned}
C_{m}\left\|u_{h, p}-u_{2 G}\right\|_{\mathrm{DG}}^{2} & \leq A_{h, p}^{\prime}\left[u_{H, P}\right]\left(u_{h, p}-u_{2 G}, \phi\right) \\
& =A_{h, p}^{\prime}\left[u_{H, P}\right]\left(u_{h, p}-u_{H, P}, \phi\right)+A_{h, p}^{\prime}\left[u_{H, P}\right]\left(u_{H, P}-u_{2 G}, \phi\right) \\
& =A_{h, p}^{\prime}\left[u_{H, P}\right]\left(u_{h, p}-u_{H, P}, \phi\right)+A_{h, p}\left(u_{H, P}, \phi\right)-F_{h, p}(\phi) \\
& =A_{h, p}^{\prime}\left[u_{H, P}\right]\left(u_{h, p}-u_{H, P}, \phi\right)+A_{h, p}\left(u_{H, P}, \phi\right)-A_{h, p}\left(u_{h, p}, \phi\right) \\
& =-\mathcal{Q}\left(u_{H, P}, u_{h, p}, \phi\right) .
\end{aligned}
$$


Hence, from Lemma 3.3 we get that

$$
\left\|u_{h, p}-u_{2 G}\right\|_{\mathrm{DG}} \leq C p^{2} h^{-1}\left(1+\left\|\nabla u_{h, p}\right\|_{L^{\infty}(\Omega)}+\left\|\nabla u_{H, P}\right\|_{L^{\infty}(\Omega)}\right)\left\|u_{h, p}-u_{H, P}\right\|_{\mathrm{DG}}^{2} .
$$

Applying Lemma 3.4, noting that $p^{3 / 2} \geq P^{3 / 2} \geq 1$, and the a priori bound for the standard IP DGFEM, cf. [6, Theorem 3.3], gives

$$
\begin{aligned}
\left\|u_{h, p}-u_{2 G}\right\|_{\mathrm{DG}} & \leq C p^{2} h^{-1}\left(1+p^{3 / 2}+P^{3 / 2}\right)\left\{\left\|u-u_{h, p}\right\|_{\mathrm{DG}}^{2}+\left\|u-u_{H, P}\right\|_{\mathrm{DG}}^{2}\right\} \\
& \leq C p^{7 / 2} h^{-1}\left\{\frac{h^{2 s-2}}{p^{2 k-3}}\|u\|_{H^{k}(\Omega)}^{2}+\frac{H^{2 S-2}}{P^{2 k-3}}\|u\|_{H^{k}(\Omega)}^{2}\right\} .
\end{aligned}
$$

Noting that $h \leq H$ and $p \geq P$ completes the proof of the first bound (3.1). To prove the second inequality (3.2), we first employ the triangle inequality

$$
\left\|u-u_{2 G}\right\|_{\mathrm{DG}} \leq\left\|u-u_{h, p}\right\|_{\mathrm{DG}}+\left\|u_{h, p}-u_{2 G}\right\|_{\mathrm{DG}} .
$$

Thereby, applying the a priori error bound for the standard IP DGFEM, together with the bound (3.1), completes the proof of Theorem 3.1.

\section{A Posteriori Error Analysis}

Here, we state an a posteriori error bound for the two-grid IP DGFEM.

TheOREM 4.1. Let $u \in \mathrm{H}_{0}^{1}(\Omega)$ be the analytical solution of (1.1), $u_{H, P} \in$ $V\left(\mathcal{T}_{H}, \boldsymbol{P}\right)$ and $u_{2 G} \in V\left(\mathcal{T}_{h}, \boldsymbol{p}\right)$ the numerical approximations obtained from (2.3) and (2.4), respectively; then the following $h p$-a posteriori error bound holds

$$
\left\|u-u_{2 G}\right\|_{\mathrm{DG}}^{2} \leq C \sum_{\kappa \in \mathcal{T}_{h}}\left(\eta_{\kappa}^{2}+\xi_{\kappa}^{2}\right),
$$

with a constant $C>0$, which is independent of $h, H, \boldsymbol{p}$ and $\boldsymbol{P}$. Here, for $\kappa \in \mathcal{T}_{h}$,

$$
\begin{aligned}
\eta_{\kappa}^{2}= & h_{\kappa}^{2} p_{\kappa}^{-2}\left\|\Pi_{\kappa, p_{\kappa}} f+\nabla \cdot\left\{\mu\left(\left|\nabla u_{H, P}\right|\right) \nabla u_{2 G}\right\}\right\|_{L^{2}(\kappa)}^{2} \\
& +h_{e} p_{e}^{-1}\left\|\llbracket \mu\left(\left|\nabla u_{H, P}\right|\right) \nabla u_{2 G} \rrbracket\right\|_{L^{2}(\partial \kappa \backslash \Gamma)}^{2}+\gamma^{2} h_{e}^{-1} p_{e}^{3}\left\|\llbracket u_{2 G} \rrbracket\right\|_{L^{2}(\partial \kappa)}^{2}, \\
\xi_{\kappa}^{2}= & \left\|\left(\mu\left(\left|\nabla u_{H, P}\right|\right)-\mu\left(\left|\nabla u_{2 G}\right|\right)\right) \nabla u_{2 G}\right\|_{L^{2}(\kappa)}^{2} \\
& +\left\|\left(\mu_{\nabla u}^{\prime}\left(\left|\nabla u_{H, P}\right|\right) \cdot\left(\nabla u_{2 G}-\nabla u_{H, P}\right)\right) \nabla u_{H, P}\right\|_{L^{2}(\kappa)}^{2} \\
& +h_{e} p_{e}^{-1}\left\|\left(\mu_{\nabla u}^{\prime}\left(\left|\nabla u_{H, P}\right|\right) \cdot\left(\nabla u_{2 G}-\nabla u_{H, P}\right)\right) \nabla u_{H, P}\right\|_{L^{2}(\partial \kappa)}^{2},
\end{aligned}
$$

and $\Pi_{\kappa, p_{\kappa}}$ denotes the (elementwise) $L^{2}$-projection onto $V\left(\mathcal{T}_{h}, \boldsymbol{p}\right)$.

Proof. The proof of this error bound follows in an analogous manner to the a posteriori proof presented in [5], cf. also [7]. For details, we refer to $[\mathbf{3}]$.

\section{Numerical Experiments}

In this section we perform numerical experiments to validate the a priori error bound, Theorem 3.1 and demonstrate the performance of the a posteriori error bound, Theorem 4.1; here, we set $\gamma=10$ and $\theta=0$. Throughout this section, we let $\Omega$ be the unit square $(0,1)^{2} \subset \mathbb{R}^{2}$ and define the nonlinear coefficient as $\mu(\boldsymbol{x},|\nabla u|)=2+(1+|\nabla u|)^{-1}$. We select the right-hand forcing function $f$ so that the analytical solution to $(1.1)$ is given by $u(x, y)=x(1-x) y(1-y)(1-2 y) \mathrm{e}^{-20(2 x-1)^{2}}$. 


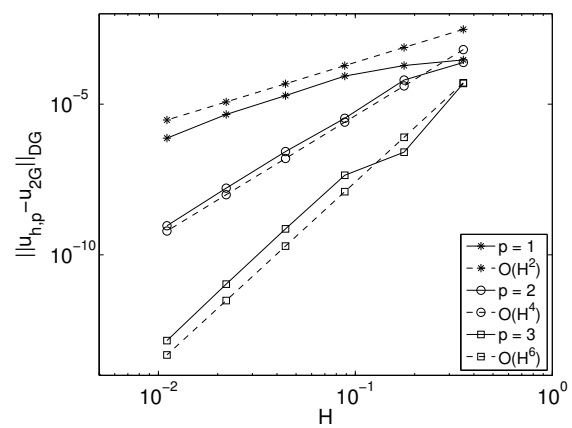

FiguRE 1. Convergence of error between $u_{2 G}$ and $u_{h, p}$.

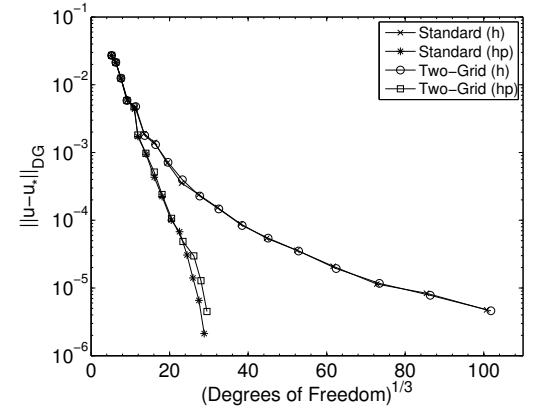

(a)

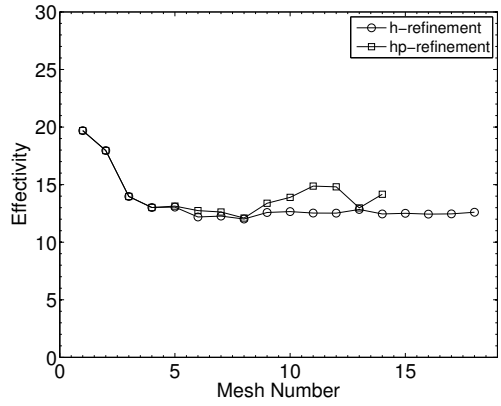

(b)

Figure 2. (a) Comparison of the error in the DGFEM norm, using both the IP DGFEM $\left(u_{*}=u_{h, p}\right)$ and the two-grid IP DGFEM $\left(u_{*}=u_{2 G}\right)$; (b) Effectivity indices of the two-grid IP DGFEM.

5.1. Validation of Theorem 3.1. We first validate the bound given in Theorem 3.1; to this end we first solve the standard IP DGFEM on a $256 \times 256$ uniform mesh of quadrilaterals to compute $u_{h, p}$ for a fixed constant polynomial degree $p=1,2,3$. We then compute the solution $u_{2 G}$ to $(2.3)-(2.4)$, for $p=1,2,3$, on a fixed fine $256 \times 256$ mesh, while performing uniform $h$-refinement of the coarse mesh, starting from a $4 \times 4$ mesh with polynomial degree $P=p$. Figure 1 shows the convergence rate of the error between $u_{h, p}$ and $u_{2 G}$, measured in the DG norm, compared to the size of the coarse mesh. Here, we observe that $\left\|u_{h, p}-u_{2 G}\right\|_{\mathrm{DG}}$ tends to zero at the optimal rate $\mathcal{O}\left(H^{2 P}\right)$, for each fixed $P$, cf. Theorem 3.1.

5.2. Adaptive Refinement using Theorem 4.1. For this experiment we use the two-grid mesh adaptation algorithm from [5], with the local error indicators $\eta_{\kappa}$ and local two-grid error indicators $\xi_{\kappa}$ from Theorem 4.1, to automatically refine the coarse and fine meshes employing both $h$ - and $h p$-adaptive mesh refinement. Figure 2 shows $\left\|u-u_{2 G}\right\|_{\mathrm{DG}}$ compared to the third root of the degrees of freedom, as well as the effectivity indices of the error estimator. As can be seen for both $h-$ and $h p$-adaptive refinement, the effectivity indices are roughly constant, indicating that the error bound overestimates the error by a roughly constant factor. For reference purposes, we also calculate the standard IP DGFEM solution $u_{h, p}$, using 

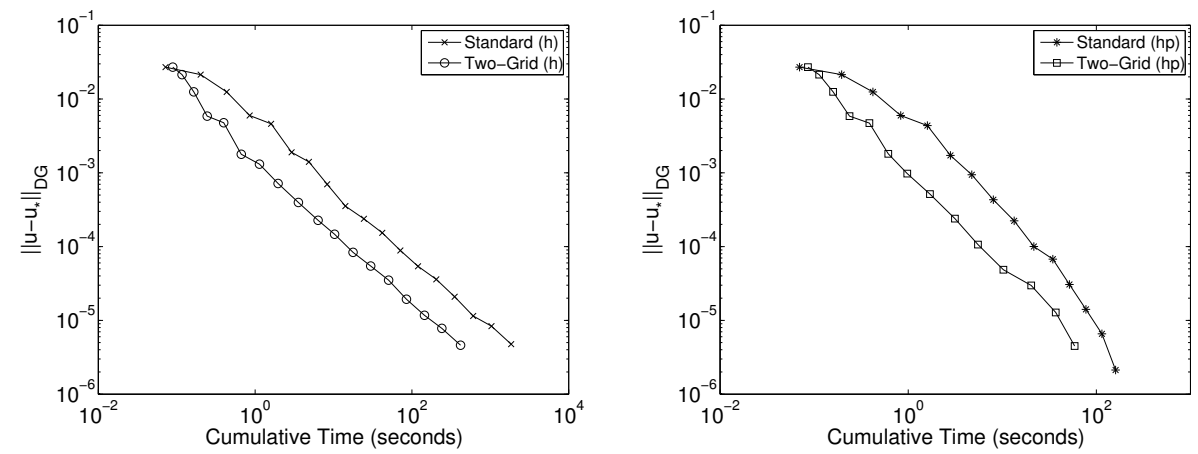

FIgURE 3 . CPU timing of the IP DGFEM $\left(u_{*}=u_{h, p}\right)$ and the two-grid IP DGFEM $\left(u_{*}=u_{2 G}\right)$ employing $h$ - and $h p$-refinement.

both $h$ - and $h p$-adaptive refinement; cf. Figure 2(a). Finally, in Figure 3 we compare the error in the standard and two-grid IP DGFEMs against the cumulative CPU time when both $h-$ and $h p$-adaptive refinement are employed; here, we observe that the two-grid IP DGFEM is more efficient than the standard IP DGFEM.

\section{References}

1. O. Axelsson and W. Layton, A two-level method for the discretization of nonlinear boundary value problems, SIAM J. Numer. Anal. 33 (1996), no. 6, 2359-2374.

2. I. Babuška and M. Suri, The $h-p$ version of the finite element method with quasiuniform meshes, RAIRO Modél. Math. Anal. Numér. 21 (1987), no. 2, 199-238.

3. S. Congreve, Discontinuous Galerkin finite element methods for quasilinear partial differential equations, Ph.D. thesis, University of Nottingham, in preparation.

4. S. Congreve, P. Houston, and T. P. Wihler, Two-grid hp-version DGFEMs for strongly monotone second-order quasilinear elliptic PDEs, Proc. Appl. Math. Mech. 11 (2011), 3-6.

5. _ Two-grid hp-version discontinuous Galerkin finite element methods for second-order quasilinear elliptic PDEs, J. Sci. Comput. (in press).

6. P. Houston, J. Robson, and E. Süli, Discontinuous Galerkin finite element approximation of quasilinear elliptic boundary value problems I: the scalar case, IMA J. Numer. Anal. 25 (2005), 726-749.

7. P. Houston, E. Süli, and T. P. Wihler, A posteriori error analysis of hp-version discontinuous Galerkin finite-element methods for second-order quasi-linear PDEs, IMA J. Numer. Anal. 28 (2008), no. 2, 245-273.

8. C. Schwab, p- and hp-FEM - theory and applications in solid and fluid mechanics, Oxford University Press, Oxford, 1998.

9. J. Xu, Two-grid discretization techniques for linear and nonlinear PDEs, SIAM J. Numer. Anal. 33 (1996), no. 5, 1759-1777.

School of Mathematical Sciences, University of Nottingham, University Park, NotTingham, NG7 2RD, UK

E-mail address: pmxsc@nottingham.ac.uk

E-mail address: Paul.Houston@nottingham.ac.uk 